Grand Valley State University

ScholarWorks@GVSU

2012

\title{
Communication: Multi-state Analysis of the OCS Ultraviolet Absorption Including Vibrational Structure
}

\author{
J. A. Schmidt \\ University of Copenhagen \\ M. S. Johnson \\ University of Copenhagen \\ George C. McBane \\ Grand Valley State University, mcbaneg@gvsu.edu \\ R. Schinke \\ Max-Planck-Institut für Dynamik und Selbstorganisation
}

Follow this and additional works at: https://scholarworks.gvsu.edu/chm_articles

\section{ScholarWorks Citation}

Schmidt, J. A.; Johnson, M. S.; McBane, George C.; and Schinke, R., "Communication: Multi-state Analysis of the OCS Ultraviolet Absorption Including Vibrational Structure" (2012). Peer Reviewed Articles. 1.

https://scholarworks.gvsu.edu/chm_articles/1

This Article is brought to you for free and open access by the Chemistry Department at ScholarWorks@GVSU. It has been accepted for inclusion in Peer Reviewed Articles by an authorized administrator of ScholarWorks@GVSU.

For more information, please contact scholarworks@gvsu.edu. 


\title{
Communication: Multi-state analysis of the OCS ultraviolet absorption including vibrational structure
}

\author{
J. A. Schmidt, ${ }^{1, a)}$ M. S. Johnson, ${ }^{1}$ G. C. McBane, ${ }^{2}$ and R. Schinke ${ }^{3, b)}$ \\ ${ }^{1}$ Department of Chemistry, University of Copenhagen, Universitetsparken 5 , \\ DK-2100 Copenhagen $\emptyset$, Denmark \\ ${ }^{2}$ Department of Chemistry, Grand Valley State University, Allendale, Michigan 49401, USA \\ ${ }^{3}$ Max-Planck-Institut für Dynamik und Selbstorganisation (MPIDS), D-37077 Göttingen, Germany
}

(Received 28 February 2012; accepted 22 March 2012; published online 5 April 2012)

\begin{abstract}
The first absorption band of OCS (carbonyl sulfide) is analyzed using potential energy surfaces and transition dipole moment functions of the lowest four singlet and the lowest four triplet states. Excitation of the $2^{1} A^{\prime}$ state is predominant except at very low photon energies. It is shown that the vibrational structures in the center of the band are due to excitation of the $2^{3} A^{\prime \prime}$ triplet state, whereas the structures at very low energies are caused by bending excitation in the potential wells of states $2{ }^{1} A^{\prime}$ and $1{ }^{1} A^{\prime \prime}$. () 2012 American Institute of Physics. [http://dx.doi.org/10.1063/1.3701699]
\end{abstract}

Carbonyl sulfide (OCS) is the most abundant sulfur compound in the atmosphere and has a significant affect on atmospheric chemistry. UV photolysis of OCS in the stratosphere is the main source of stratospheric sulfate. ${ }^{1}$ Many laboratory studies ${ }^{2-6}$ have been performed to unravel the apparently complex photodissociation dynamics of OCS in the first absorption band $(222 \mathrm{~nm}))^{7,8}$ Despite the numerous experiments several open questions remain, including the role of the ${ }^{1} A^{\prime \prime}$ states ${ }^{5}$ and the origin of the vibrational structures of the absorption spectrum. The very few existing theoretical analyses ${ }^{3,9}$ were helpful to explain the coarse dissociation dynamics, but a comprehensive understanding in terms of quantum dynamics on accurate potential energy surfaces (PES) including several states is overdue.

OCS has 16 valence electrons and is isoelectronic with $\mathrm{CO}_{2}$ and $\mathrm{N}_{2} \mathrm{O}$. The photodissociation of the latter has been comprehensively unraveled by means of quantum mechanical calculations using global PESs and transition dipole moment (TDM) functions. ${ }^{10-14}$ The low-lying electronic states and the corresponding PESs of OCS (Refs. 3 and 9) are very similar to those of $\mathrm{N}_{2} \mathrm{O}$ (Ref. 10) and so are the dissociation dynamics: The fragmentation is fast and proceeds mainly via the bent $2^{1} A^{\prime}$ state with high rotational excitation of the molecular fragment. ${ }^{2,3}$ Nevertheless, there are significant differences which only full-dimensional quantum mechanical calculations on several PESs are able to resolve.

This communication is the first report of a theoretical study on the UV photodissociation of OCS. The focus here is the absorption spectrum, namely the contributions of the various excited states and the vibrational structures at the center of the band around $220 \mathrm{~nm}$ (Ref. 8) and at its onset around $280 \mathrm{~nm}^{7}$ We show that these structures have different electronic and vibrational origins than in the electronic spectrum of $\mathrm{N}_{2} \mathrm{O}$.

We calculated global three-dimensional PESs for the four lowest singlet states (letters in parentheses denote abbrevia-

\footnotetext{
a)Electronic mail: johanalbrechtschmidt@gmail.com.

b)Electronic mail: rschink@ gwdg.de.
}

tions used below, see Table I) $-1{ }^{1} A^{\prime}(\mathrm{X}), 2{ }^{1} A^{\prime}(\mathrm{A}), 1{ }^{1} A^{\prime \prime}(\mathrm{B})$, and $2{ }^{1} A^{\prime \prime}(\mathrm{C}),-$ and the four lowest triplet states $-1{ }^{3} A^{\prime}$ (a), $2^{3} A^{\prime}(\mathrm{d}), 1^{3} A^{\prime \prime}(\mathrm{b})$, and $2^{3} A^{\prime \prime}$ (c) — at basically the same level of electronic structure theory as for $\mathrm{N}_{2} \mathrm{O}$ (Refs. 10 and 13): multi-configuration reference internally contracted configuration interaction (MRCI) theory based on wave functions obtained by state-averaged full valence complete active space self consistent field (CASSCF) calculations. In the MRCI calculations, the number of active and core orbitals were 12 and 7, respectively. The augmented correlation consistent polarized valence quadruple zeta (aug-cc-pVQZ) basis set was used. The Jacobi coordinates $R$ (distance from $\mathrm{S}$ to the center of mass of $\mathrm{CO}$ ), $r$ (CO bond length), and $\gamma$ (angle between $\mathbf{R}$ and $\mathbf{r}$ ) were used. The grid for the PESs was defined by $3.2 a_{0} \leq R \leq 10 a_{0}, 1.8 a_{0} \leq r \leq 3.6 a_{0}\left(1.9 a_{0} \leq r \leq 2.6 a_{0}\right.$ for the triplet states), and $0 \leq \gamma \leq 180^{\circ}$. In what follows, energies are normalized with respect to the equilibrium of the $\mathrm{X}$ state.

In addition to the PESs, the TDMs with the ground state $\mathrm{X}$ were also calculated at the MRCI level of electronic structure theory. Spin-orbit (SO) coupling was necessarily taken into account in the calculation of the TDMs of the triplet states. The sum over the three TDMs of the individual SO states for each triplet state was considered. ${ }^{13}$ All electronic structure calculations were performed with the MOLPRO suite of programs. ${ }^{15}$

Absorption cross sections were calculated separately for all of the seven excited states by three-dimensional wave packet calculations in the time domain. ${ }^{11}$ The initial wave packet at $t=0$ in the excited state was defined as a product of a vibrational wave function in the ground state and the modulus of the transition dipole moment function with the absorbing electronic state. The absorption cross sections were determined by Fourier transformation of the autocorrelation functions. ${ }^{16}$ Temperature dependent cross sections were calculated by Boltzmann averaging over initial states $(0,0,0),(0,1,0),(0,2,0),(0,3,0),(1,0,0)$, and $(1,1,0)$. As in the calculations for $\mathrm{N}_{2} \mathrm{O},{ }^{10,11}$ the total angular momentum was zero and the non-adiabatic couplings between the states 
TABLE I. Excited electronic states included in the analysis.

\begin{tabular}{llccccc}
\hline \hline$C_{s}$ & $C_{\infty v v}$ & Abbrev. & Type & $E_{\mathrm{v}}{ }^{\mathrm{a}}$ & $\mathrm{TDM}^{\mathrm{b}}$ & $\sigma_{\max }{ }^{\mathrm{c}}$ \\
\hline $2^{1} A^{\prime}$ & $1^{1} \Delta$ & $\mathrm{A}$ & disso. & 5.82 & $5.7(-2)^{\mathrm{d}}$ & 1.73 \\
$1^{1} A^{\prime \prime}$ & $1^{1} \Sigma^{-}$ & $\mathrm{B}$ & disso. & 5.73 & $1.4(-2)$ & $7.26(-2)$ \\
$2{ }^{1} A^{\prime \prime}$ & $1^{1} \Delta$ & $\mathrm{C}$ & bound & 5.88 & $7.1(-3)$ & $9.30(-3)$ \\
$1^{3} A^{\prime}$ & $1^{3} \Sigma^{+}$ & $\mathrm{a}$ & disso. & 5.05 & $9.3(-4)$ & $2.92(-4)$ \\
$2{ }^{3} A^{\prime}$ & $1^{3} \Delta$ & $\mathrm{d}$ & bound & 5.45 & $2.7(-3)$ & $3.35(-3)$ \\
$1^{3} A^{\prime \prime}$ & $1^{3} \Delta$ & $\mathrm{b}$ & disso. & 5.42 & $5.8(-3)$ & $9.06(-3)$ \\
$2{ }^{3} A^{\prime \prime}$ & $1^{3} \Sigma^{-}$ & $\mathrm{c}$ & bound & 5.76 & $3.1(-2)$ & $1.29(-1)$ \\
\hline
\end{tabular}

${ }^{a}$ Vertical excitation energy $E_{\mathrm{V}}(\mathrm{eV})$ at $R=4.2 a_{0}, r=2.2 a_{0}$, and $\gamma=5^{\circ}$.

${ }^{\mathrm{b}}$ Transition dipole moments $|\mu|$ (atomic units) at $R=4.2 a_{0}, r=2.2 a_{0}$, and $\gamma=5^{\circ}$

${ }^{\mathrm{c}}$ Absorption cross section at the maximum in units $10^{-19} \mathrm{~cm}^{2}$.

${ }^{\mathrm{d}}$ Numbers in parentheses indicate powers of ten

(see below) were neglected. Details of the electronic structure and the dynamics calculations will be published elsewhere.

Figure 1 shows cuts through the lowest four singlet and lowest four triplet PESs vs. dissociation coordinate $R$. The cusps for the adiabatic potentials $\mathrm{C}$ and $\mathrm{c}$ are caused by avoided crossings with higher states of the same symmetry. The singlet states correlate with the singlet channel $\operatorname{CO}\left({ }^{1} \Sigma^{+}\right)$ $+\mathrm{S}\left({ }^{1} D\right)$ while the triplet states $\mathrm{a}, \mathrm{b}$, and c correlate with the triplet channel $\mathrm{CO}\left({ }^{1} \Sigma^{+}\right)+\mathrm{S}\left({ }^{3} P\right)$. States $\mathrm{A}$ and $\mathrm{C}$ and states $\mathrm{b}$ and $\mathrm{d}$ are degenerate at the OCS linear configuration and form Renner-Teller pairs (Table I). States B and C interact via nonadiabatic coupling and the same applies to $\mathrm{a} / \mathrm{d}$ and $\mathrm{b} / \mathrm{c}$. In principle, all states are also coupled by SO coupling, which is larger than for $\mathrm{N}_{2} \mathrm{O}$.

All seven excited states depicted in Fig. 1 have excitation energies in the range of the first absorption band. Table I summarizes the vertical energies $E_{\mathrm{v}}$ and the TDMs at an approximate Franck-Condon (FC) point close to the ground state equilibrium. Also tabulated are the maximum absorption cross sections $\sigma_{\max }$ calculated for initial state $(0,0,0)$; the
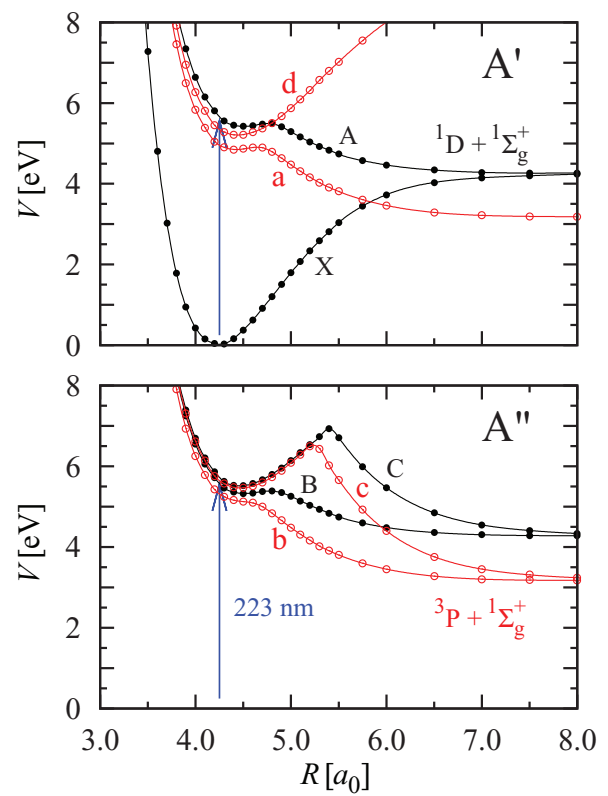

FIG. 1. Cuts through the lowest singlet (black) and triplet (red) PESs for $r=2.2 a_{0}$ and $\gamma=5^{\circ}$. The arrow illustrates excitation at $223 \mathrm{~nm}$, the center of the band.
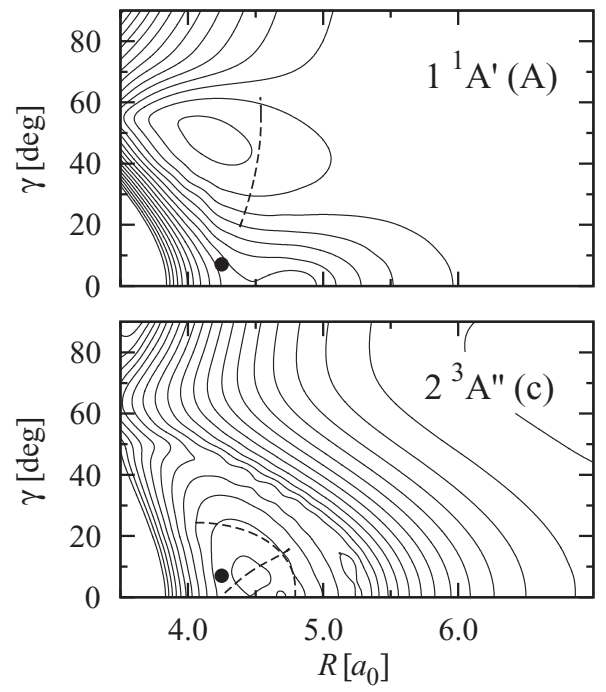

FIG. 2. Contour representations of the A- and the c-state PESs for fixed $r$ $=2.20 a_{0}$. The highest contour is $8 \mathrm{eV}$ and the spacing is $0.25 \mathrm{eV}$. The dots mark the approximate Franck-Condon point and the dashed lines represent classical periodic orbits for $E=4.5 \mathrm{eV}$ (upper panel) and $6 \mathrm{eV}$ (lower panel).

data for the bound states were obtained from low-resolution spectra. Excitation of the A state makes by far the main contribution. The cross section of B is about 25 times smaller at the maximum and plays only a minor role. Excitation of the B state becomes significant only at very low photon energies, where its contribution eventually exceeds that of the A state (see below). This finding is in accord with the analysis of Brouard et al. ${ }^{5}$ of the anisotropy parameter $\beta$ at several wavelengths.

Most notable is the large TDM and therefore the large cross section of the bound triplet state c. Close to linearity $(\gamma$ $<7^{\circ}$ ), c and A are almost degenerate which leads to strong mixing through the SO interaction ${ }^{13}$ and a significant peak of the c-state TDM near $5^{\circ}$. Beyond this maximum it rapidly diminishes.

In Fig. 2, we show contour representations of the two most important excited states, A (largest cross section) and c (origin of vibrational structures), as functions of $R$ and $\gamma$. Like the corresponding PES for $\mathrm{N}_{2} \mathrm{O}$, the A-state PES has a bent potential minimum around $48^{\circ}$; the energy is slightly below the (classical) dissociation threshold. The B-state PES has a very similar shape; its minimum occurs at slightly smaller angles. The $\mathrm{c}$ state has its minimum near linearity with a low barrier at $\gamma=0$. It consists of a bound part and a repulsive part, separated by a high barrier caused by the avoided crossing with a higher ${ }^{3} A^{\prime \prime}$ PES. Unlike $\mathrm{N}_{2} \mathrm{O}$, the gradient of the $\mathrm{A}$ and $\mathrm{B}$ PESs with respect to the $\mathrm{CO}$ stretching coordinate is very small, near the FC point as well as along the entire fragmentation path.

The absorption cross sections for state A do not show any vibrational structures, neither for $(0,0,0)$ shown in Fig. 3, nor for the other initial states. All initial-state resolved cross sections for $\mathrm{B}$ are also structureless. This result came as a surprise since the corresponding cross sections for $\mathrm{N}_{2} \mathrm{O}$ do show such structures. ${ }^{10,17}$ The A and B-state PESs of OCS have the same general topography as the corresponding PESs 


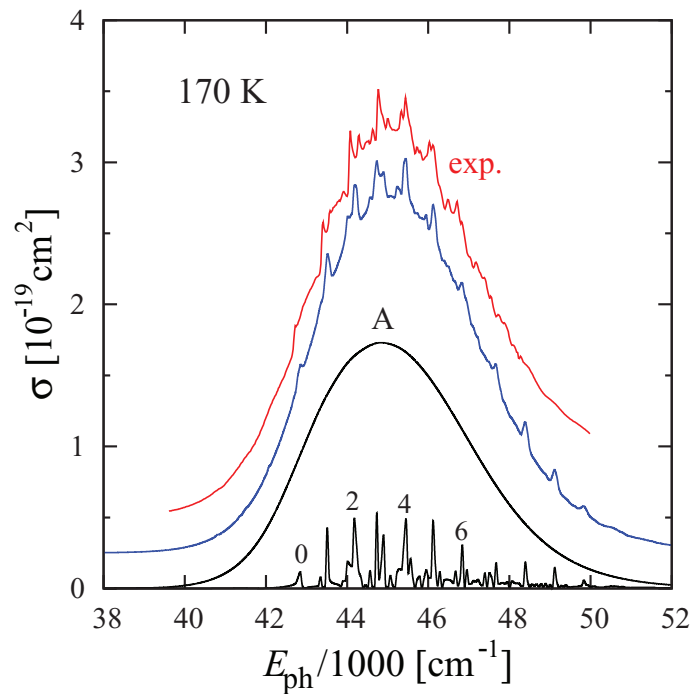

FIG. 3. Comparison of the measured cross section ${ }^{8}$ (red) and the calculated total cross section (blue, summed over all seven electronic states and scaled by 1.3 ) for $170 \mathrm{~K}$; they are shifted upward by 0.5 and 0.25 units, respectively. Also shown are the (unscaled) cross sections of states A and $\mathrm{c}$ and initial state $(0,0,0)$; the corresponding B-state cross section is not presented because of its small amplitude. The numbers over the peaks of the c-state cross section refer to the polyad quantum numbers $P$.

of $\mathrm{N}_{2} \mathrm{O}$, so the differences in the absorption spectra must be caused by differences in the detailed fragmentation dynamics. A small part of the $\mathrm{N}_{2} \mathrm{O}$ wave packet is temporarily trapped by the bent potential well around $50^{\circ}$, which leads to a small recurrence in the autocorrelation function. ${ }^{16}$ However, simultaneous elongation of the $\mathrm{NN}$ bond is the prerequisite because the well depth and thus the probability for being trapped increases with $r$; the cross section for fixed $r$ is structureless. It is the combined large-amplitude bending and NN stretching motion that causes the recurrence and consequently the weak vibrational structures in the $\mathrm{N}_{2} \mathrm{O}$ spectrum. In the dissociation of OCS, on the other hand, the CO vibrational mode is merely a spectator and the $\mathrm{CO}$ vibrational distribution is very cold. At energies near the maximum of the spectrum the probability of $\mathrm{CO}(v=1)$ is $\sim 0.2$ in accord with experiment. ${ }^{2}$ The lack of CO stretching causes the wave packet to bypass the large- $r$ region of the PES where the barrier to dissociation is largest and therefore the trapping is most efficient.

The triplet state $2^{3} A^{\prime \prime}$ (c) is binding and excitation of its vibrational states leads to a long progression as illustrated in Fig. 3 for $(0,0,0)$. The spectrum is organized in terms of polyads $P=0,1, \ldots$ with $P+1$ states per polyad. The progression starts at $E_{\mathrm{ph}} \approx 42850 \mathrm{~cm}^{-1}$; for comparison, the first weak structure in the $170 \mathrm{~K}$ spectrum $^{8}$ appears at $42725 \mathrm{~cm}^{-1}$ (Fig. 3). The three fundamental frequencies (in $\mathrm{cm}^{-1}$ ) are 522 (bending, for $K=0$ ), 688 (OC-S stretch), and 1711 (CO stretch). The bending and OC-S stretching modes are strongly mixed and form the polyads. Representative classical periodic orbits (PO) shown in Fig. 2 illustrate the underlying motions. Excitation of the CO stretch is not apparent in the spectrum. The spacing between the main peaks, averaged over the central part of the spectrum, is about $660 \mathrm{~cm}^{-1}$ and agrees perfectly with the main spacing in the measured spectrum.

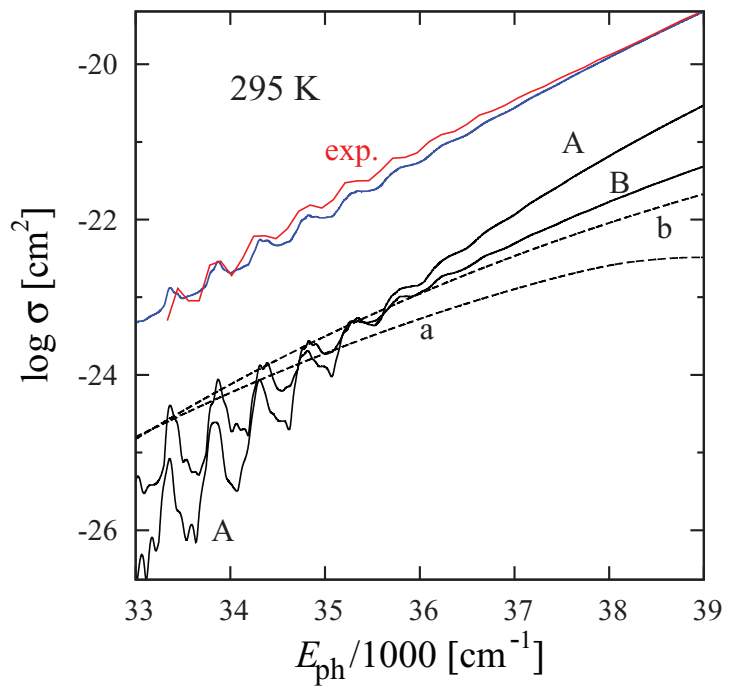

FIG. 4. Comparison of the measured ${ }^{7}$ (red) and the calculated (blue) absorption cross sections for $295 \mathrm{~K}$ in the low-energy tail (multiplied by 10 for clarity). Also shown are the individual $295 \mathrm{~K}$ cross sections of the singlet states A and B (solid lines) and the triplet states a and $\mathrm{b}$ (dashed lines).

Because the TDM of the c state is relatively large the vibrational structures clearly show up in the total cross section obtained by summation over all seven electronic states. The widths and therefore the intensities of the c-state cross section are affected by coupling to the nearby dissociative states as well as incoherent rotational broadening. These effects were not included in the calculations, but were qualitatively accounted for by averaging over a moving Gaussian with a full width at half maximum of $125 \mathrm{~cm}^{-1}$. All cross sections for electronic state A were shifted by $200 \mathrm{~cm}^{-1}$ to higher energies, while the cross sections for all other electronic states were not shifted. Finally, the total cross section was scaled by an overall factor of 1.3 (1.37 for $\mathrm{N}_{2} \mathrm{O}$ (Ref. 10)). After these modifications the calculated thermal cross section for $170 \mathrm{~K}$ agrees satisfactorily with the measured spectrum ${ }^{8}$ including the vibrational structures (Fig. 3). The calculations also yield structures in the high-energy tail, which, however, are absent in the experimental spectrum.

The $2{ }^{1} A^{\prime \prime}(\mathrm{C})$ state is also bound and yields a structured spectrum similar to that of the c state. However, its calculated TDM is too small to account for the pronounced vibrational structures.

At very low excitation energies, where the absorption cross section is about four orders of magnitude smaller than at the maximum, the OCS spectrum shows another progression of vibrational structures ${ }^{7}$ (Fig. 4). These structures are reproduced by the singlet states A and B and reflect excitation of the bound and quasibound states supported by the potential wells around $48^{\circ}$ and $38^{\circ}$, respectively. The equilibrium angles in X, on one hand, and A and B on the other hand, are very different and therefore the FC overlap is extremely small. However, bending excitation in the ground state and/or in the excited states exponentially increases the overlap and thus the cross section. With increasing energy, the lifetime of the resonance states decreases, the structures become broader, and the intensities gradually diminish. 
The origin of this progression is at $E_{\mathrm{ph}} \approx 31000 \mathrm{~cm}^{-1}$ for $\mathrm{A}$ and $\approx 31100 \mathrm{~cm}^{-1}$ for $\mathrm{B}$ and the fundamental frequencies are $602 \mathrm{~cm}^{-1}$ and $590 \mathrm{~cm}^{-1}$, respectively. The essential motion is illustrated by the PO in Fig. 2. The bending mode has a relatively large anharmonicity and therefore the energy spacings decrease to about $480 \mathrm{~cm}^{-1}$ in the region of $34000 \mathrm{~cm}^{-1}$, compared to $\approx 450 \mathrm{~cm}^{-1}$ in the measured spectrum. Because the bending frequency in the $\mathrm{X}$ state, $516 \mathrm{~cm}^{-1}$, is similar to the bending frequencies of $\mathrm{A}$ and $\mathrm{B}$, hot bands are coincident with the $(0,0,0)$ absorption maxima; Boltzmann averaging merely leads to some weak broadening when the temperature is increased. ${ }^{7}$ Although the TDM for state B is much smaller than that for A, the B-state cross section is of similar size at very low energies. The ratio is about $1: 1$ around $35000 \mathrm{~cm}^{-1}$ (corresponding to $286 \mathrm{~nm}$ ) as suggested by Brouard et al. ${ }^{5}$ Because the repulsive triplet states a and $b$ have excitation energies significantly below the A state, they exceed the singlet cross sections below $34000 \mathrm{~cm}^{-1}$ and partly fill up the gaps between the peaks. The calculated $295 \mathrm{~K}$ absorption cross section in the very red tail of the spectrum agrees very well with the measured one, concerning both the absolute value and the vibrational structures. The cross sections in Fig. 4 were not shifted on the energy axis; however, the calculated thermal cross section was multiplied by 1.3 as in Fig. 3. The low-energy tail of the OCS absorption band is reminiscent of the Huggins band of ozone. ${ }^{18}$

The present study provides the first realistic account of the $220 \mathrm{~nm}$ absorption spectrum of OCS. The calculations show that: (1) The main vibrational structures are due to excitation of the bound $2^{3} A^{\prime \prime}$ triplet state. (2) The structures at very low energies reflect bending excitation in the bent potential wells of $2^{1} A^{\prime}$ and $1^{1} A^{\prime \prime}$. The calculations also shed light on a long-lasting debate: (3) Except for very long wavelengths, excitation of state $1^{1} A^{\prime \prime}$ is insignificant.

${ }^{1}$ C. Brühl, J. Lelieveld, P. J. Crutzen, and H. Tost, Atmos. Chem. Phys. 12, 1239 (2012)

${ }^{2}$ N. Sivakumar, G. E. Hall, P. L. Houston, J. W. Hepburn, and I. Burak, J. Chem. Phys. 88, 3692 (1988).

${ }^{3}$ T. Suzuki, H. Katayanagi, S. Nanbu, and M. Aoyagi, J. Chem. Phys. 109, 5778 (1998).

${ }^{4}$ T. P. Rakitzis, A. J. van den Brom, and M. H. M. Janssen, Science 303, 1852 (2004)

${ }^{5}$ M. Brouard, A. V. Green, F. Quadrini, and C. Vallance, J. Chem. Phys. 127, 084304 (2007).

${ }^{6}$ S. Hattori, S. O. Danielache, M. S. Johnson, J. A. Schmidt, H. G. Kjaergaard, S. Toyoda, Y. Ueno, and N. Yoshida, Atmos. Chem. Phys. 11, 10293 (2011).

${ }^{7}$ L. T. Molina, J. J. Lamb, and M. J. Molina, Geophys. Res. Lett. 8, 1008, doi:10.1029/GL008i009p01008 (1981).

${ }^{8}$ C. Y. R. Wu, F. Z. Chen, and D. L. Judge, J. Quant. Spectrosc. Radiat. Transf. 61, 265 (1999).

${ }^{9}$ S. O. Danielache, S. Nanbu, C. Eskebjerg, M. S. Johnson, and N. Yoshida, J. Chem. Phys. 131, 024307 (2009).

${ }^{10}$ R. Schinke, J. Chem. Phys. 134, 064313 (2011).

${ }^{11}$ J. A. Schmidt, M. S. Johnson, U. Lorenz, G. C. McBane, and R. Schinke, J. Chem. Phys. 135, 024311 (2011).

${ }^{12}$ J. A. Schmidt, M. S. Johnson, and R. Schinke, Atmos. Chem. Phys. 11, 8965 (2011).

${ }^{13}$ R. Schinke, J. A. Schmidt, and M. S. Johnson, J. Chem. Phys. 135, 194303 (2011).

${ }^{14}$ G. C. McBane and R. Schinke, J. Chem. Phys. 136, 044314 (2012).

${ }^{15}$ H.-J. Werner, P. J. Knowles, R. Lindh, F. R. Manby, M. Schütz, et al., MOLPRO, version 2006.1, a package of ab initio programs, 2006, see http://www.molpro.net.

${ }^{16}$ R. Schinke, Photodissociation Dynamics (Cambridge University Press, Cambridge, 1993).

${ }^{17}$ R. Schinke, J. Suarez, and S. C. Farantos, J. Chem. Phys. 133, 091103 (2010).

${ }^{18}$ S. Y. Grebenshchikov, Z.-W. Qu, H. Zhu, and R. Schinke, Phys. Chem. Chem. Phys. 9, 2044 (2007). 